\title{
Genomic Sequence Resource of Kabatiella zeae, the Causative Pathogen of Corn Eyespot Disease
}

\author{
Yafei Wang, ${ }^{1,2}$ Jinai Yao, ${ }^{1,3}$ Xinyao Xia, ${ }^{1}$ Zhiqiang Li, ${ }^{1}$ Shaoqun Zhou, ${ }^{2}$ Wende Liu, ${ }^{1, \dagger}$ and \\ Hanxiang $\mathrm{Wu}^{1, \dagger}$ \\ ${ }^{1}$ State Key Laboratory for Biology of Plant Diseases and Insect Pests, Institute of Plant Protection, \\ Chinese Academy of Agricultural Sciences, 100193, Beijing, China \\ ${ }^{2}$ Shenzhen Branch, Guangdong Laboratory for Lingnan Modern Agriculture, Genome Analysis \\ Laboratory of the Ministry of Agriculture, Agricultural Genomics Institute at Shenzhen, Chinese \\ Academy of Agricultural Sciences, 440307, Shenzhen, China \\ ${ }^{3}$ Fujian Key Laboratory for Monitoring and Integrated Management of Crop Pests/Institute of Plant \\ Protection, Fujian Academy of Agricultural Sciences, 350013, Fuzhou, China
}

\section{Abstract}

Kabatiella zeae is the causative pathogen of corn eyespot disease, which is an important leaf disease that damages corn (Zea mays L.) worldwide. In this study, we provided an annotated draft of the assembled genome of the $K$. zeae field strain KZ1 through PacBio and Illumina sequencing. The assembled KZ1 genome size is $23,602,820 \mathrm{bp}$, and its GC content is $50.71 \%$. The completeness of the assembled genome is $97.6 \%$ in this study. The assembly obtained in this study has 94 contigs and the length of $N_{50}$ is $720,243 \mathrm{bp}$. This study is the first report of the $K$. zeae genome, which contributes to further research on the genetic variation and pathogenic mechanism of this important fungal pathogen.

Corn eyespot disease, which is caused by Kabatiella zeae, is one of the most serious leaf diseases of corn (Zea mays L.) worldwide. K. zeae belongs to order Tuberculariales, class Hyphomycetes, genus Kabatiella. It has been pointed out that Kabatiella was synonymous with Aureobasidium in 1973, and Aureobasidium zeae was identified as the pathogen of corn eyespot disease based on fungal classification rules (Dingley 1973). According to morphological and molecular biology studies, Kabatiella was considered to be a different genus from Aureobasidium; therefore, the pathogenic fungus of corn eyespot was reidentified as Kabatiella zeae in 2011 (Seifert and Gams 2011). Corn eyespot disease was first discovered in Japan in 1959 (Narita and Hiratsuka 1959), then successively found in most corn-growing areas around the world (Arny et al. 1971; Reifschneider and Arny 1979; Santos et al. 2007; Shurtle 1980; Sun et al. 2016). Corn eyespot disease was reported in Jilin Province of China in 1964 (Qi et al. 1966). In 1998, the disease caused a serious loss of total crop yield on farms in Liaoning Province of China (Xu et al. 2000). Thereafter, the disease gradually spread to corn-growing areas in northeast, north, and northwest China, resulting in serious losses in corn production. Corn eyespot disease has recently attracted the attention of researchers because of its serious outbreaks in certain corn-growing areas of China (Sun et al. 2016; Wang et al. 2014; Zhang 2014). However, there is currently no information about the genome of $K$. zeae, which limits the prevention and control of corn eyespot disease. The emergence of new

${ }^{\dagger}$ Corresponding authors: W. Liu; liuwende@ caas.cn and H. Wu; wuhanxiang@caas.cn

*The $e$-Xtra logo stands for "electronic extra" and indicates that a supplementary figure is published online.

The author(s) declare no conflict of interest.

Accepted for publication 14 June 2021. $e-$ tra $^{*}$

\section{Funding}

This work was financially supported by grants from the Agricultural Science and Technology Innovation Program and Shenzhen Science and Technology Program (grant number KQTD20180411143628272).

\section{Keywords}

genome, genomics, Illumina sequencing, Kabatiella zeae, PacBio sequencing, pathogenesis 
Table 1. Genome assembly statistics of Kabatiella zeae field isolate KZ1

\begin{tabular}{ll} 
Variables & Statistics \\
Genome assembly size (bp) & $23,602,820$ \\
Number of contigs & 94 \\
Contig N50 (bp) & 720,243 \\
Contig No (bp) & 158,685 \\
Largest contig length (bp) & $1,560,680$ \\
GC content (\%) & 50.71 \\
BUSCO completeness (\%) & 97.6 \\
Number of genes & 8,064 \\
Number of virulence genes & 920 \\
Number of CAZymes & 341 \\
Number of transmembrane proteins & 954 \\
Number of transport proteins & 1,346 \\
Number of secreted proteins & 639 \\
\hline
\end{tabular}

whole-genome sequencing technologies provides a powerful tool for high-quality genome assembly of important pathogenic fungi (Huo et al. 2021; Zhu et al. 2019). Here, we use PacBio sequencing technology to present the first draft of the genome assembly for $K$. zeae and analyze its genome characteristics, which will help in understanding the pathogenic mechanism of this fungus and developing integrated approaches to control corn eyespot disease.

Strain KZ1 was isolated from field corn leaves in Gongzhuling, Jilin Province, China in October 2019, cultured with potato dextrose agar medium, then subjected to single-spore isolation. Combined with internal transcribed spacer gene sequence analysis and morphological characteristics shown in Supplementary Figure S1, KZ1 was identified as K. zeae. The purified fresh fungal tissues were collected for whole-genome sequencing. The Omega Fungal DNA Kit D3390-02 was used to extract fungal genomic DNA, a TBS-380 fluorometer (Turner BioSystems Inc., Sunnyvale, CA, U.S.A.) was used to evaluate the purity and content of DNA, and $0.8 \%$ agarose gel electrophoresis was used to test the integrity of the extracted DNA. The PacBio Sequel Single Molecule Real-Time (SMRT) sequencing platform was used to perform whole-genome sequencing of high-quality DNA samples. The SMRTbell library was prepared using the sheared genomic DNA through the SMRTbell sequencing adapter (Pacific Biosciences), and an insert library of approximately $10 \mathrm{~kb}$ was sequenced on an SMRT cell using standard methods. The low-quality and short subreads were removed; then, the qualified PacBio subreads were assembled using nextDenovo v2.0-beta.1 (Aimé et al. 2013). All settings were the default except for the following: genome size $=20 \mathrm{M}$, expected seed depth $=100$, and seed cutoff $=6,129$. The NEXTflex rapid DNA-Seq kit was used to prepare Illumina sequencing libraries and perform pair-end sequencing on the Illumina HiSeq 4000 platform (parameters were set to $150 \mathrm{bp}$ ). The obtained Illumina sequencing data were used to polish the genome assembled with PacBio data through Pilon software (Walker et al. 2014) with default settings and, finally, 8,697 errors were corrected (Walker et al. 2014). The completeness of the assembled genome sequence was evaluated by BUSCO v3, with the gene set shown by Simão et al. (2015). MAKER2 v2.31.9 software was used to predict the gene model in the assembled genome (Holt and Yandell 2011). The parameters were set as follows: organism type = eukaryotic, cpus $=10$, max dna len $=100,000$, min contig $=1$, pred stats $=1$, min protein $=30$, map forward $=0$, keep preds $=1$, split hit $=4,000$, min intron $=20$, single exon $=1$, single length $=250$, correct est fusion $=0$, and evaluate $=1$. Basic annotations for the predicted genes were made through five major databases, including Pfam, Swiss-Prot, Gene Ontology, NonRedundant Protein Database, and Clusters of Orthologous Groups of Proteins (Ashburner et al. 2000; Boutet et al. 2007; El-Gebali et al. 2019; Pruitt et al. 2005; Tatusov et al. 2000). The Kyoto Encyclopedia of Genes and Genomes database was used to further systematically annotate the predicted genes (Kanehisa and Goto 2000).

In this study, PacBio SMRT sequencing produced 2,438,457,264 bp (sequencing depth $103 x$ ) and Illumina paired-end sequencing produced 6,308,846,440 bp (sequencing depth $267 x$ ) of raw read data. The size of the assembled KZ1 genome was $23,602,820 \mathrm{bp}$, and the GC content of the genome was $50.71 \%$. The completeness of the assembled KZ1 genome was $97.6 \%$. In this study, 94 contigs were assembled, and $\mathrm{N}_{50}$ was 720,243 bp. Among them, the 
contig 44 (JAGFJS010000044.1) was thought to be the mitochondrial genome of KZ1 after NCBI blast. More characteristics of the assembled genome are shown in Table 1. We used MAKER2 software to predict fungal genes and obtained 8,064 genes. Barrnap v0.8 and tRNAscan-SE v1.3.1 software with default settings were used to predict the ribosomal RNA (rRNA) and transfer RNA (tRNA) contained in the genome, and finally obtained 36 rRNAs and 169 tRNAs, respectively (Lowe and Eddy 1997). Carbohydrate-active enzymes (CAZymes) are usually closely related to fungal pathogenicity and affect the interaction between fungi and their hosts (van den Brink and de Vries 2011). The CAZymes identified in the assembled genome by searching against the CAZy database include 182 glycoside hydrolases, 61 glycosyltransferases, 49 auxiliary activities, 46 carbohydrate esterases, 2 polysaccharide lyases, and 1 carbohydrate-binding module (Cantarel et al. 2009). Cytochrome P450 enzymes are a large family of proteins with heme as a prosthetic group. They participate in the metabolism of many endogenous and exogenous substances, including drugs and environmental compounds. In this study, we identified a total of 127 genes in the cytochrome P450 family. It is worth noting that 920 virulence genes related to pathogenesis were predicted through the Database of Fungal Virulence Factors (Lu et al. 2012). After searching against the Pathogen Host Interaction database (E-value cutoff = 1e-05), 2,395 related genes were identified, in which 12 genes were identified as encoding potential effectors (Winnenburg et al. 2006). In addition, 954 transmembrane proteins were identified by searching against the TMHMM database, 1,346 transport proteins were identified by searching against the TCDB database, and 639 secreted proteins were identified by searching against the SignalP database (E-value cutoff $=1 \mathrm{e}-05$ for all searches) (Krogh et al. 2001; Petersen et al. 2011; Saier et al. 2006). These genes are involved in the pathogenic process of fungi and may play important roles in the pathogenesis of $K$. zeae.

We used RepeatMasker v4.0.7 software with default settings to predict the repetitive sequences in the KZ1 genome, and further classified the identified repetitive sequences (Chen 2004). Interspersed repeats, also known as transposon elements (TEs), usually affect the pathogenic variation of various plant fungi (Faino et al. 2016; Raffaele and Kamoun 2012). The total length of the identified repetitive sequence was $11,343 \mathrm{bp}$, which accounted for $0.05 \%$ of the assembled whole genome. We identified 84 LINE retrotransposons, 24 SINE retrotransposons, 18 DNA transposons, 10 LTR retrotransposons, and 1 unclassified TE in the $\mathrm{KZ1}$ genome. In addition, by searching for the telomere repeats at both ends of each assembled contig, telomere repeats (TTAGGG) were finally identified at the $3^{\prime}$ ends of contigs 57 (JAGFJS010000057.1) and 94 (JAGFJS010000094.1).

The KZ1 genome assembly provided here is the first available genome of $K$. zeae, which can improve our understanding of the genome characteristics of $K$. zeae and its pathogenic mechanism. To our knowledge, the $\mathrm{KZ1}$ genome assembled here is also the first available genome of the Kabatiella genus. The strain sequenced in this study is stored in the State Key Laboratory for Biology of Plant Diseases and Insect Pests, Institute of Plant Protection, Chinese Academy of Agricultural Sciences, Beijing, China. PacBio and Illumina sequencing read data have been submitted to NCBI, and the accession number is PRJNA719577. This Whole Genome Shotgun project has been deposited at GenBank under the accession JAGFJS000000000. The version described in this article is version JAGFJS020000000.

Annotation files, supporting files, and the genome analysis result files containing more information are available in the Zenodo repository.

\section{Author-Recommended Internet Resources}

Barrnap: https://github.com/tseemann/barrnap

BUSCO: https://busco.ezlab.org

MAKER2: http://www.yandell-lab.org/software/maker.html

RepeatMasker: http://www.repeatmasker.org

Zenodo: https://zenodo.org/record/4777356

\section{Literature Cited}

Aimé, S., Alabouvette, C., Steinberg, C., and Olivain, C. 2013. The endophytic strain Fusarium oxysporum Fo47: A good candidate for priming the defense responses in tomato roots. Mol. Plant-Microbe Interact. 26:918-926.
Arny, D. C., Smalley, E. B., Ullstrup, A. J., Worf, G. L., and Ahrens, R. W. 1971. Eyespot of maize, a disease new to North America. Phytopathology 61:54-57. Ashburner, M., Ball, C. A., Blake, J. A., Botstein, D., Butler, H., Cherry, J. M., Davis, A. P., Dolinski, K., Dwight, S. S., Eppig, J. T., Harris, M. A., Hill, D. P., 
Issel-Tarver, L., Kasarskis, A., Lewis, S., Matese, J. C., Richardson, J. E., Ringwald, M., Rubin, G. M., Sherlock, G., and The Gene Ontology Consortium. 2000. Gene ontology: Tool for the unification of biology. Nat. Genet. 25:25-29.

Boutet, E., Lieberherr, D., Tognolli, M., Schneider, M., and Bairoch, A. 2007. Uniprotkb/Swiss-Prot. Pages 89-112 in: Plant Bioinformatics. Humana Press, Totowa, NJ, U.S.A.

Cantarel, B. L., Coutinho, P. M., Rancurel, C., Bernard, T., Lombard, V., and Henrissat, B. 2009. The carbohydrate-active enzymes database (CAZy): An expert resource for glycogenomics. Nucleic Acids Res. 37:D233-D238.

Chen, N. 2004. Using RepeatMasker to identify repetitive elements in genomic sequences. Curr. Protoc. Bioinf. 5:4.10.1-4.10.14.

Dingley, J. 1973. Eyespot disease of maize in New Zealand. N. Z. J. Agric. Res. 16 : 325-328.

El-Gebali, S., Mistry, J., Bateman, A., Eddy, S. R., Luciani, A., Potter, S. C., Qureshi, M., Richardson, L. J., Salazar, G. A., Smart, A., Sonnhammer, E. L. L., Hirsh, L., Paladin, L., Piovesan, D., Tosatto, S. C. E., and Finn, R. D. 2019. The Pfam protein families database in 2019. Nucleic Acids Res. 47:D427-D432.

Faino, L., Seidl, M. F., Shi-Kunne, X., Pauper, M., van den Berg, G. C., Wittenberg, A. H., and Thomma, B. P. 2016. Transposons passively and actively contribute to evolution of the two-speed genome of a fungal pathogen. Genome Res. 26:1091-1100.

Holt, C., and Yandell, M. 2011. MAKER2: An annotation pipeline and genome-database management tool for second-generation genome projects. BMC Bioinf. 12:491.

Huo, J., Wang, Y., Hao, Y., Yao, Y., Wang, Y., Zhang, K., Tan, X., Li, Z., and Wang, W. 2021. Genome sequence resource for Colletotrichum scovillei, the cause of anthracnose disease of chili. Mol. Plant-Microbe Interact. 34:122-126.

Kanehisa, M., and Goto, S. 2000. KEGG: Kyoto Encyclopedia of Genes and Genomes. Nucleic Acids Res. 28:27-30.

Krogh, A., Larsson, B., von Heijne, G., and Sonnhammer, E. L. L. 2001. Predicting transmembrane protein topology with a hidden Markov model: Application to complete genomes. J. Mol. Biol. 305:567-580.

Lowe, T. M., and Eddy, S. R. 1997. tRNAscan-SE: A program for improved detection of transfer RNA genes in genomic sequence. Nucleic Acids Res. 25:955-964.

Lu, T., Yao, B., and Zhang, C. 2012. DFVF: Database of fungal virulence factors. Database (Oxford) 2012:bas032.

Narita, T., and Hiratsuka, Y. 1959. Studies on Kabatiella zeae n. sp. the causal fungus of a new leaf spot disease of corn. Jpn. J. Phytopathol. 24:147-153.

Petersen, T. N., Brunak, S., von Heijne, G., and Nielsen, H. 2011. SignalP 4.0: Discriminating signal peptides from transmembrane regions. Nat. Methods 8: 785-786.

Pruitt, K. D., Tatusova, T., and Maglott, D. R. 2005. NCBI Reference Sequence (RefSeq): A curated non-redundant sequence database of genomes, transcripts and proteins. Nucleic Acids Res. 33:D501-D504.

Qi, P. K., Bai, J. K., and Zhu, G. X. 1966. Pages 28-30 in: Cultivated Plant Fungal Diseases in Jilin Province. Science Press, Beijing, China.
Raffaele, S., and Kamoun, S. 2012. Genome evolution in filamentous plant pathogens: Why bigger can be better. Nat. Rev. Microbiol. 10:417-430.

Reifschneider, F. J. B., and Arny, D. C. 1979. Seed infection of maize (Zea mays) by Kabatiella zeae. Plant Dis. Rep. 63:352-354.

Saier, M. H. J., Jr., Tran, C. V., and Barabote, R. D. 2006. TCDB: The Transporter Classification Database for membrane transport protein analyses and information. Nucleic Acids Res. 34:D181-D186.

Santos, I. D., Silva, A. D., and Malagi, G. 2007. Ocorrência de mancha ocular em milho causada por Kabatiella zeae no Paraná e em Santa Catarina [Occurrence of maize eyespot caused by Kabatiella zeae in Paraná and Santa Catarina state, Brazil]. Fitopatol. Bras. 32:359.

Seifert, K. A., and Gams, W. 2011. The genera of Hyphomycetes-2011 update. Persoonia 27:119-129.

Shurtle, M. C. 1980. Pages 21-22 in: Compendium of Corn Diseases. American Phytopathological Society, St. Paul, MN, U.S.A.

Simão, F. A., Waterhouse, R. M., loannidis, P., Kriventseva, E. V., and Zdobnov, E. M. 2015. BUSCO: Assessing genome assembly and annotation completeness with single-copy orthologs. Bioinformatics 31:3210-3212.

Sun, J. Y., Xiao, S. Q., Xu, J. N., Lu, K. X., Xue, C. S., and Chen, J. 2016. Occurrence condition and chemical control of maize eyespot in Liaoning Province. J. Maize Sci. 24:147-151.

Tatusov, R. L., Galperin, M. Y., Natale, D. A., and Koonin, E. V. 2000. The COG database: A tool for genome-scale analysis of protein functions and evolution. Nucleic Acids Res. 28:33-36.

van den Brink, J., and de Vries, R. P. 2011. Fungal enzyme sets for plant polysaccharide degradation. Appl. Microbiol. Biotechnol. 91:1477-1492.

Walker, B. J., Abeel, T., Shea, T., Priest, M., Abouelliel, A., Sakthikumar, S., Cuomo, C. A., Zeng, Q., Wortman, J., Young, S. K., and Earl, A. M. 2014. Pilon: An integrated tool for comprehensive microbial variant detection and genome assembly improvement. PLoS One 9:e112963.

Wang, D., He, D., Li, G., Gao, S., Lv, H., Shan, Q., and Wang, L. 2014. An efficient tool for random insertional mutagenesis: Agrobacterium tumefaciens-mediated transformation of the filamentous fungus Aspergillus terreus. J. Microbiol. Methods 98:114-118.

Winnenburg, R., Baldwin, T. K., Urban, M., Rawlings, C., Köhler, J., and Hammond-Kosack, K. E. 2006. PHI-base: A new database for pathogen host interactions. Nucleic Acids Res. 34:D459-D464.

Xu, X. D., Dong, H. Y., Jiang, Y., Qiao, Y., Liu, Z. H., and Sun, J. D. 2000. Preliminary studies on the northern anthracnose disease in maize. J. Shenyang Agric. Univ. 31:507-510.

Zhang, Q. F. 2014. The corn northern anthracnose. Sci. Technol. W. China 13:4950 .

Zhu, B., Wang, S., Mi, C. Y., Yang, R. H., Zen, G. H., and Hu, X. F. 2019. Genome sequence resource for llyonectria mors-panacis, causing rusty root rot of Panax notoginseng. Mol. Plant-Microbe Interact. 32:1468-1471. 